\title{
Identification of potential biomarkers in cholestasis and the therapeutic effect of melatonin by metabolomics, multivariate data and pathway analyses
}

\author{
HAN YU $^{1 *}$, YUNZHOU LI $^{1 *}$, ZONGYING XU ${ }^{1 *}$, DINGNAN WANG ${ }^{1}$, SHAOHUA SHI ${ }^{1}$, HUIFANG DENG $^{1}$, \\ BAIHUI ZENG ${ }^{1}$, ZHILI ZHENG $^{2}$, LILI SUN ${ }^{1}$, XIULAN DENG $^{2}$ and XIANGGEN ZHONG ${ }^{1}$ \\ ${ }^{1}$ Synopsis of Golden Chamber and ${ }^{2}$ Pharmacology Departments, Chinese Medicine College, \\ Beijing University of Chinese Medicine, Chaoyang, Beijing 100029, P.R. China
}

Received March 18, 2018; Accepted September 4, 2018

DOI: $10.3892 /$ ijmm.2018.3859

\begin{abstract}
The present study investigated the anti-cholestatic effect of melatonin (MT) against $\alpha$-naphthyl isothiocyanate (ANIT)-induced liver injury in rats and screened for potential biomarkers of cholestasis. Rats were administered ANIT by intraperitoneal injection and then sacrificed $36 \mathrm{~h}$ later. Serum biochemical parameters were measured and liver tissue samples were subjected to histological analysis. Active components in the serum were identified by gas chromatography-mass spectrometry, while biomarkers and biochemical pathways were identified by multivariate data analysis. The results revealed that the serum levels of alanine aminotransferase, aspartate aminotransferase, total bilirubin, direct
\end{abstract}

Correspondence to: Professor Xianggen Zhong, Synopsis of Golden Chamber Department, Chinese Medicine College, Beijing University of Chinese Medicine, 11 North 3rd Ring East Road, Chaoyang, Beijing 100029, P.R. China

E-mail: zhongxg@bucm.edu.cn

Professor Xiulan Deng, Pharmacology Department, Chinese Medicine College, Beijing University of Chinese Medicine, 11 North 3rd Ring East Road, Chaoyang, Beijing 100029, P.R. China

E-mail: dengxiulan@bucm.edu.cn

*Contributed equally

Abbreviations: ALP, alkaline phosphatase; ALT, alanine aminotransferase; ANIT, $\alpha$-naphthyl isothiocyanate; AST, aspartate aminotransferase; BSTFA, $N, O$-bis(trimethylsilyl)trifluoroacetamide; CMC, carboxymethyl cellulose sodium salt; DBIL, direct bilirubin; GC-MS, gas chromatography-mass spectrometry; GGT, $\gamma$-glutamyl transpeptidase; HE, hematoxylin-eosin; KEGG, Kyoto Encyclopedia of Genes and Genomes; MT, melatonin; OPLS-DA, orthogonal partial least-squares discriminant analysis; PCA, principle component analysis; QC, quality control; TBIL, total bilirubin; TMCS, trimethylchlorosilane; UDCA, ursodeoxycholic acid; VIP, variable influence on projection

Key words: metabolomics, cholestasis, melatonin, pathway analysis bilirubin, $\gamma$-glutamyl transpeptidase, and alkaline phosphatase were reduced in rats with ANIT-induced cholestasis that were treated with MT. The histological observations indicated that MT had a protective effect against ANIT-induced hepatic tissue damage. Metabolomics analysis revealed that this effect was likely to be associated with the regulation of compounds related to MT synthesis and catabolism, and amino acid metabolism, including 5-aminopentanoate, 5-methoxytryptamine, L-tryptophan, threonine, glutathione, L-methionine, and indolelactate. In addition, principal component analysis demonstrated that the levels of these metabolites differed significantly between the MT and control groups, providing further evidence that they may be responsible for the effects induced by MT. These results provide an insight into the mechanisms underlying cholestasis development and highlight potential biomarkers for disease diagnosis.

\section{Introduction}

Cholestasis is characterized by a reduction in bile flow and bile acid accumulation (1), and a higher incidence of this condition is observed in hepatopathy. A previous study in Shanghai revealed that the total incidence of cholestasis was $10.26 \%$ among chronic liver disease patients (2). Cholestasis is classified as intrahepatic or extrahepatic (3), and mechanisms associated with the former type can be broadly classified as hepatocellular or obstructive. Plugging of interlobular bile ducts, portal expansion and bile duct proliferation, along with centrilobular cholate injury, are observed in the obstructive subtype $(3,4)$. Cholestasis can be caused by pre-existing medical conditions including infections, drug treatments, and metabolic or genetic disorders (5); therefore, it is considered as a secondary disease (6). Without appropriate treatment, liver cells (such as portal myofibroblasts and hepatic stellate cells) are hyperactivated, leading to biliary fibrosis or even cirrhosis $(7,8)$. To date, there are few therapeutic options available for the treatment of cholestasis (9).

Ursodeoxycholic acid (UDCA) is currently the only drug approved by the U.S. Food and Drug Administration for cholestasis treatment, which acts by relieving disease symptoms and restoring liver enzyme levels (10). However, 
approximately one-third of patients present little or no response to UDCA therapy $(11,12)$. Melatonin (MT) is a methoxyindole synthesized and secreted at night by the pineal gland under normal light/dark conditions $(13,14)$. It is widely distributed in human tissues (13) and serves an important role in a number of physiological processes $(15,16)$. For instance, MT exerts an anti-inflammatory effect by scavenging reactive oxygen species and inhibiting lipid peroxidation (13,17-19). In addition, oral administration of MT was reported to abolish the increase in the total cholesterol concentration in the serum of rats with $\alpha$-naphthyl isothiocyanate (ANIT)-induced acute liver injury and cholestasis by restoring cholesterol metabolism and transport in the liver (20). However, few studies have investigated the effects of orally administered MT on serum metabolome profiles in rats with cholestasis.

Metabolomics analysis has been widely used to evaluate the therapeutic effects of herbal medicines (21), as it provides a global view of low molecular weight metabolites in biological samples, reflecting physiological changes in biochemical networks and signaling pathways. It has been suggested that the development of cholestasis may be associated with alterations in endogenous metabolite profiles (22). Metabolomics has previously been applied to investigate the therapeutic effects of Paeonia lactiflora Pall (23), yinchenhao (24) and rhubarb (25) as treatment approaches for cholestasis.

ANIT is a well-known hepatotoxicant that causes bile duct epithelial cells to release factors that attract neutrophils, leading to hepatic injury (26-30). ANIT-induced intrahepatic cholestasis shares similarities with drug-induced cholangiolitic hepatitis in humans. Transient intrahepatic cholestasis can be induced in animal models with a single dose of ANIT, which has been demonstrated in rats (30-34) as well as mice and guinea pigs (31).

The present study investigated the anti-cholestatic effect of MT in rats. Gas chromatography-mass spectrometry (GC-MS) analysis was conducted to evaluate the metabolite profiles, while multivariate data analysis was performed in order to identify biomarkers and biochemical signaling pathways associated with cholestasis.

\section{Materials and methods}

Materials and reagents. Carboxymethyl cellulose sodium salt (CMC) was obtained from Yuanye Biological Technology Co., Ltd. (Shanghai, China). ANIT and MT were purchased from Sigma-Aldrich (Merck KGaA; Darmstadt, Germany). ANIT was dissolved in olive oil and administered at a dose of $75 \mathrm{mg} / \mathrm{kg}$ body weight [1 $\mathrm{ml}$ ANIT solution in olive oil $(75 \mathrm{mg} / \mathrm{ml})$ per $100 \mathrm{~g}$ body weight] to induce liver injury with cholestasis (35). MT (100 mg/kg body weight) was resuspended in $1 \mathrm{ml}$ of $0.25 \% \mathrm{CMC}(20)$. Colorimetric assay kits were purchased to determine serum alkaline phosphatase (ALP; cat. no. A059-1), aspartate aminotransferase (AST; cat. no. C010-2), alanine aminotransferase (ALT; cat. no. C0009-2) and $\gamma$-glutamyl transpeptidase (GGT; cat. no. C017-1) levels. total bilirubin (TBIL; cat. no. C019-1), direct bilirubin (DBIL; cat. no. C019-2) and were detected by chemical oxidation assays. All assay kits were purchased from Nanjing Jiancheng Bioengineering Institute (Nanjing, China). Methanol, acetonitrile, methoxylamine hydro- chloride, n-hexane, pyridine and $N, O$-bis(trimethylsilyl) trifluoroacetamide (BSTFA) with 1\% trimethylchlorosilane (TMCS) were purchased from CNW Technologies GmbH (Düsseldorf, Germany). In addition, 2-chloro-1-phenylalanine was obtained from Shanghai Hengchuang Biotech Co., Ltd. (Shanghai, China). All other chemicals were of analytical grade and were purchased from commercial sources.

Animals and treatments. A total of 30 male Sprague-Dawley rats (7-8 weeks old; weighing 260 $20 \mathrm{~g}$ ) were obtained from SPF-JD-SPF Biotech Co., Ltd. (Beijing, China; certification no. SCXK-JING 2016-0002). All animals were allowed to acclimate for 1 week prior to experiments, were maintained at a constant temperature $\left(25 \pm 2^{\circ} \mathrm{C}\right)$ and $50 \%$ humidity with a 12:12-h light/dark cycle, and had free access to water and food. The study protocol was in strict accordance with the recommendations of the Guidelines for the Care and Use of Laboratory Animals of the Ministry of Science and Technology of China, and was approved by Beijing University of Chinese Medicine Medical and Experimental Animal Ethics Committee (Beijing, China).

Table I presents the experimental design employed for the animal studies. The animals were randomly divided into three groups of 10 rats each, including the control, model and MT groups. The rats were fasted for $12 \mathrm{~h}$ prior to injections. Rats in the control group were injected with the vehicle (olive oil), while the other two groups received an intraperitoneal injection of ANIT at a dose of $75 \mathrm{mg} / \mathrm{kg}$ body weight. The rats were injected with the same volume of vehicle or ANIT. Rats in the MT group were orally administered MT $(100 \mathrm{mg} / \mathrm{kg}$ body weight) $12 \mathrm{~h}$ after the initial ANIT injection. The model and control groups received the same volume of $0.25 \% \mathrm{CMC}$ at $12 \mathrm{~h}$ after the initial injection. Each rat was weighed prior to treatment with intraperitoneal injections and oral administration.

Sample collection and liver function assays. The rats were provided with standard chow and water following the completion of the treatments. Rats were then fasted for $12 \mathrm{~h}$ prior to being sacrificed at $36 \mathrm{~h}$ after the initial ANIT or vehicle injection. Blood samples were collected from the inferior vena cava, and the liver was removed from each rat immediately after sacrifice. The blood samples were collected and centrifuged at $3,500 \mathrm{x} \mathrm{g}$ and $4^{\circ} \mathrm{C}$ for $15 \mathrm{~min}$ to obtain the serum. Sterile, hemolysis-free serum samples were stored at $-80^{\circ} \mathrm{C}$ prior to determination of biochemical parameters and metabolomics analysis. All serum samples were used within 1 month. Serum ALT, AST, TBIL, DBIL, GGT and ALP levels were measured with commercial test kits according to the manufacturer's protocol.

Histological analysis of liver damage. Liver tissues were excised and fixed in $10 \%$ phosphate-buffered formalin. Fixed issues were cut into $1 \times 1 \times 0.3 \mathrm{~cm}$ sections. Sections were dehydrated in a gradient alcohol series, and embedded in paraffin wax blocks. The embedded wax blocks were fixed to the slicer and were cut into 4-5 $\mu \mathrm{m}$ thick slices. Following dewaxing slides in xylene, The slides were dipped into hematoxylin and agitated for $30 \mathrm{sec}$, rinsed in $\mathrm{H}_{2} \mathrm{O}$ for $1 \mathrm{~min}$, followed by staining with $1 \%$ eosin Y solution for $30 \mathrm{sec}$ with 
Table I. Details of the animal experimental design.

Treatment at each time point

\begin{tabular}{lccccc}
\cline { 2 - 5 } Group & $0 \mathrm{~h}$ & $12 \mathrm{~h}$ & $24 \mathrm{~h}$ & $36 \mathrm{~h}$ & $48 \mathrm{~h}$ \\
\hline Control & Fasting & Olive oil & $0.25 \% \mathrm{CMC}$ & Fasting & Sacrifice \\
Model & Fasting & $75 \mathrm{mg} / \mathrm{kg}$ ANIT & $0.25 \% \mathrm{CMC}$ & Fasting & Sacrifice \\
MT & Fasting & $75 \mathrm{mg} / \mathrm{kg}$ ANIT & $100 \mathrm{mg} / \mathrm{kg} \mathrm{MT}$ & Fasting & Sacrifice
\end{tabular}

MT, melatonin; ANIT, $\alpha$-naphthyl isothiocyanate; CMC, carboxymethyl cellulose sodium salt.

agitation, all at room temperature $\left(20-25^{\circ} \mathrm{C}\right)$. Slides were examined under a BX53 microscope (Olympus Corporation, Tokyo, Japan).

Sample preparation for metabolome profiling. Serum samples stored at $-80^{\circ} \mathrm{C}$ were thawed at room temperature, and then $50 \mu \mathrm{l}$ of each serum sample was added to a $1.5-\mathrm{ml}$ Eppendorf tube with $10 \mu \mathrm{l}$ of 2-chloro-L-phenylalanine $(0.3 \mathrm{mg} / \mathrm{ml})$ dissolved in methanol as an internal standard. The tube was vortexed for $10 \mathrm{sec}$, and $150 \mu \mathrm{l}$ of an ice-cold mixture of methanol and acetonitrile $(2: 1, \mathrm{v} / \mathrm{v})$ was added, followed by vortexing for $1 \mathrm{~min}$ and ultrasonication at an ambient temperature $\left(25-28^{\circ} \mathrm{C}\right)$ for $5 \mathrm{~min}$ before storage at $-20^{\circ} \mathrm{C}$ for $10 \mathrm{~min}$. The samples were then centrifuged at $12,000 \times \mathrm{g}$ and $4^{\circ} \mathrm{C}$ for $10 \mathrm{~min}$. The quality control (QC) sample was prepared by mixing aliquots of all samples to obtain a pooled sample. A $150-\mu 1$ volume of supernatant was then transferred to a glass sampling vial and vacuum-dried at room temperature. Following the addition of $80 \mu \mathrm{l}$ of $15 \mathrm{mg} / \mathrm{ml}$ methoxylamine hydrochloride in pyridine, the sample was vortexed for $2 \mathrm{~min}$ and incubated at $37^{\circ} \mathrm{C}$ for $90 \mathrm{~min}$. An 80- $\mu \mathrm{l}$ volume of BSTFA (with $1 \%$ TMCS) and $20 \mu \mathrm{l}$ $\mathrm{n}$-hexane were added to the mixture, followed by vortexing for $2 \mathrm{~min}$ and derivatization at $70^{\circ} \mathrm{C}$ for $60 \mathrm{~min}$. The samples were allowed to stand at ambient temperature for $30 \mathrm{~min}$ prior to GC-MS analysis.

GC-MS analysis. Derivatized samples were analyzed on a 7890B gas chromatograph paired with a 5977A mass-selective detector system (Agilent Technologies, Inc., Santa Clara, CA, USA). A DB-5MS fused-silica capillary column (30 m x $0.25 \mathrm{~mm} \times 0.25 \mu \mathrm{m}$; Agilent Technologies, Inc.) was used to separate the derivatives. Helium $(>99.999 \%)$ was used as the carrier gas at a constant flow rate of $1 \mathrm{ml} / \mathrm{min}$ through the column. The injector temperature was maintained at $260^{\circ} \mathrm{C}$, the injection volume was $1 \mu \mathrm{l}$ in the splitless mode, and the solvent delay time was set to $5 \mathrm{~min}$. The oven temperature was initially set at $60^{\circ} \mathrm{C}$, and was then increased to $125^{\circ} \mathrm{C}$ at a rate of $8^{\circ} \mathrm{C} / \mathrm{min}, 210^{\circ} \mathrm{C}$ at a rate of $5^{\circ} \mathrm{C} / \mathrm{min}, 270^{\circ} \mathrm{C}$ at a rate of $10^{\circ} \mathrm{C} / \mathrm{min}$ and $305^{\circ} \mathrm{C}$ at a rate of $20^{\circ} \mathrm{C} / \mathrm{min}$, and was finally held at $305^{\circ} \mathrm{C}$ for $5 \mathrm{~min}$. The temperature of the MS quadrupole and electron impact ion source were set to $150^{\circ} \mathrm{C}$ and $230^{\circ} \mathrm{C}$, respectively. The collision energy was $70 \mathrm{eV}$. MS data were acquired in full-scan mode (m/z 50-500). The QC was injected at regular intervals (every 10 samples) throughout the analytical run to obtain a set of data for assessing reproducibility.
Data pre-processing and statistical analysis. Chem Station version E.02.02.1431 software (Agilent Technologies, Inc.) was used to convert the file format (D) of raw data to a common data format. ChromaTOF version 4.34 software (LECO Corporation, St. Joseph, MI, USA) was used to analyze the data. Metabolites were identified using Fiehn databases (http://fiehnlab.ucdavis.edu/projects/fiehnlib) in ChromaTOF. After alignment with the Statistic Compare component in ChromaTOF, a raw data array (cvs) was obtained from three-dimensional datasets, including sample information, peak names, retention time, $\mathrm{m} / \mathrm{z}$ and peak intensities. A total of 952 peaks were detected in all the samples and the QC. Internal standards and any known pseudo-positive peaks (attributed to background noise, column bleeding or caused by the BSTFA derivatization procedure) were removed from the data array. The data were normalized to the total peak area of each sample and multiplied by 10,000, and peaks from the same metabolite were combined. The total number of detected metabolites was 366 .

The data were $\log _{2}$-transformed using Excel 2007 software (Microsoft Corporation, Redmond, WA, USA), with 0 peak area replaced with 0.000001 prior to transformation. The transformed data were imported into the SIMCA version 14.0 software package (Umetrics, Umeå, Sweden). Principle component analysis (PCA) and orthogonal partial least-squares discriminant analysis (OPLS-DA) were conducted to visualize the differences in the levels of metabolites between groups after mean centering and unit variance scaling. The Hotelling's $\mathrm{T}^{2}$ region (seen as an ellipse in score plots of the models) defined the $95 \%$ confidence interval of the modelled variation. Variable influence on projection (VIP) was used to rank the overall contribution of each variable to the OPLS-DA model, and variables with a VIP value of $>1$ were considered relevant to group discrimination. Default seven-round cross-validation and 200 response permutation test were applied, with one-seventh of the samples excluded from the mathematical model in each round in order to avoid overfitting.

Identification of differentially expressed metabolites. Differentially expressed metabolites were selected based on the combination of a statistically significant threshold of VIP values obtained from the OPLS-DA model and P-values from one-way analysis of variance (followed by Tukey's test) of normalized peak areas by SIMCA version 14.0 (Umetrics, Umeå, Sweden). Metabolites with VIP $>1$ and $\mathrm{P}<0.05$ were considered to be significantly differentially expressed. 

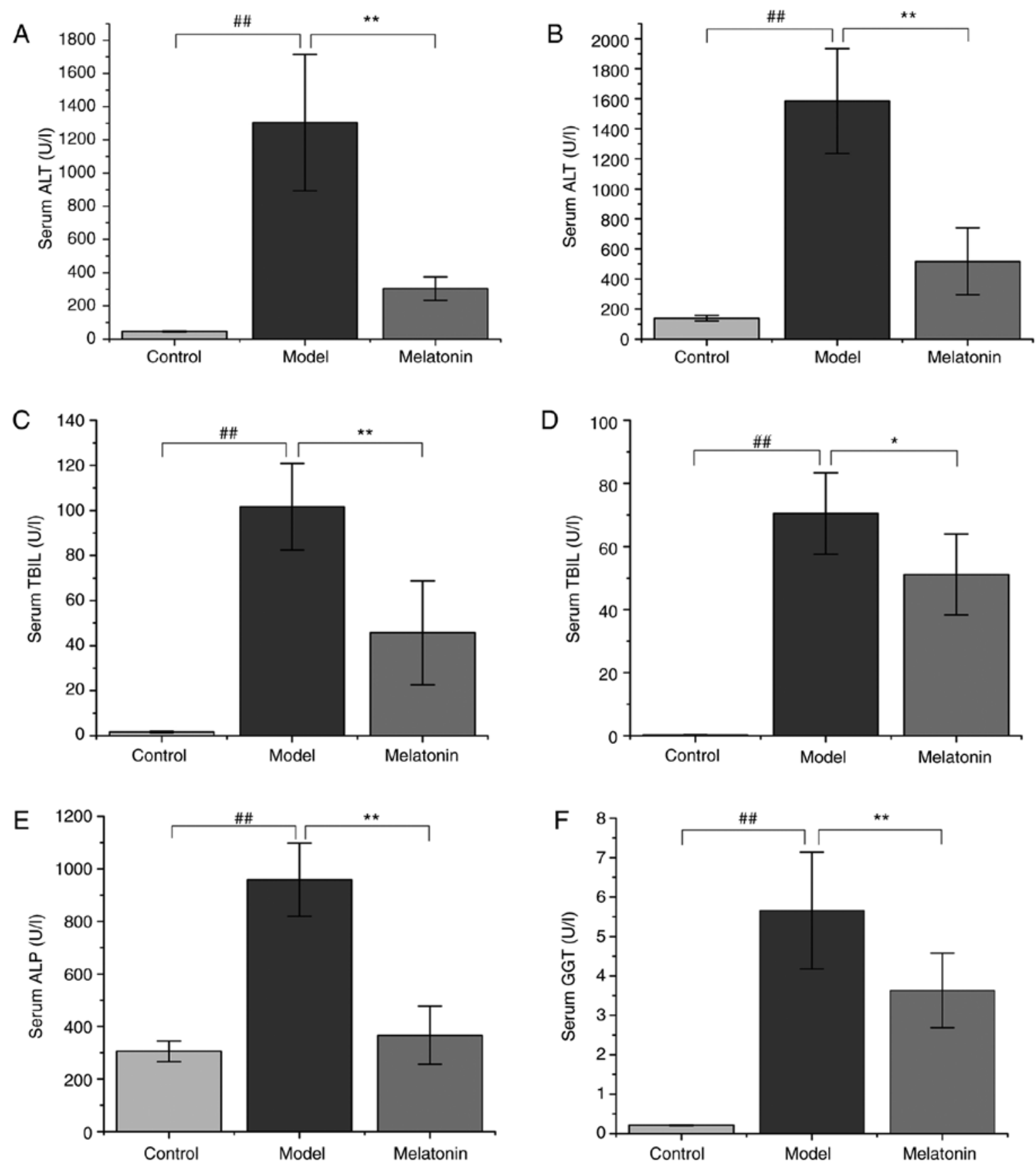

Figure 1. Effects of MT on serum biochemical parameters. Rats were treated with $\alpha$-naphthyl isothiocyanate $(75 \mathrm{mg} / \mathrm{kg}$ ) with or without MT, and liver function was assessed by measuring (A) ALT, (B) AST, (C) TBIL, (D) DBIL, (E) ALP, and (F) GGT levels. Data are expressed as the mean \pm standard error of the mean ( $\mathrm{n}=10$ per group). ${ }^{\# \#} \mathrm{P}<0.01 ;{ }^{*} \mathrm{P}<0.05$ and ${ }^{* *} \mathrm{P}<0.01$. MT, melatonin; ALT, alanine aminotransferase; AST, aspartate aminotransferase; TBIL, total bilirubin; DBIL, direct bilirubin; ALP, alkaline phosphatase; GGT, $\gamma$-glutamyl transpeptidase.

Identification of pathways. Pathways were identified with the Kyoto Encyclopedia of Genes and Genomes (https://www.kegg. $\mathrm{jp} / \mathrm{kegg} /$ ) and MBRole (http://csbg.cnb.csic.es/mbrole2/). The candidate biomarkers were mapped to KEGG by MBRole ID number shifting function. Pathways containing the candidate biomarkers were considered by KEGG pathway enrichment result.

\section{Results}

Therapeutic effect of MT on cholestasis in rats. ANIT administration resulted in significantly increased ALT and AST serum levels in rats compared with those in the control group (Fig. 1). However, treatment with $100 \mathrm{mg} / \mathrm{kg}$ MT was observed to reverse this effect (Fig. 1). Similarly, TBIL, DBIL, ALP and GGT levels were markedly increased in the ANIT group when compared with the control group. The high serum levels of these molecules indicated that ANIT successfully induced cholestasis. However, this effect was significantly reduced by MT treatment (Fig. 1).

Histological observations. Representative photomicrographs of HE-stained liver tissue from control rats and the cholestasis rats with or without MT $(100 \mathrm{mg} / \mathrm{kg})$ treatment are presented 

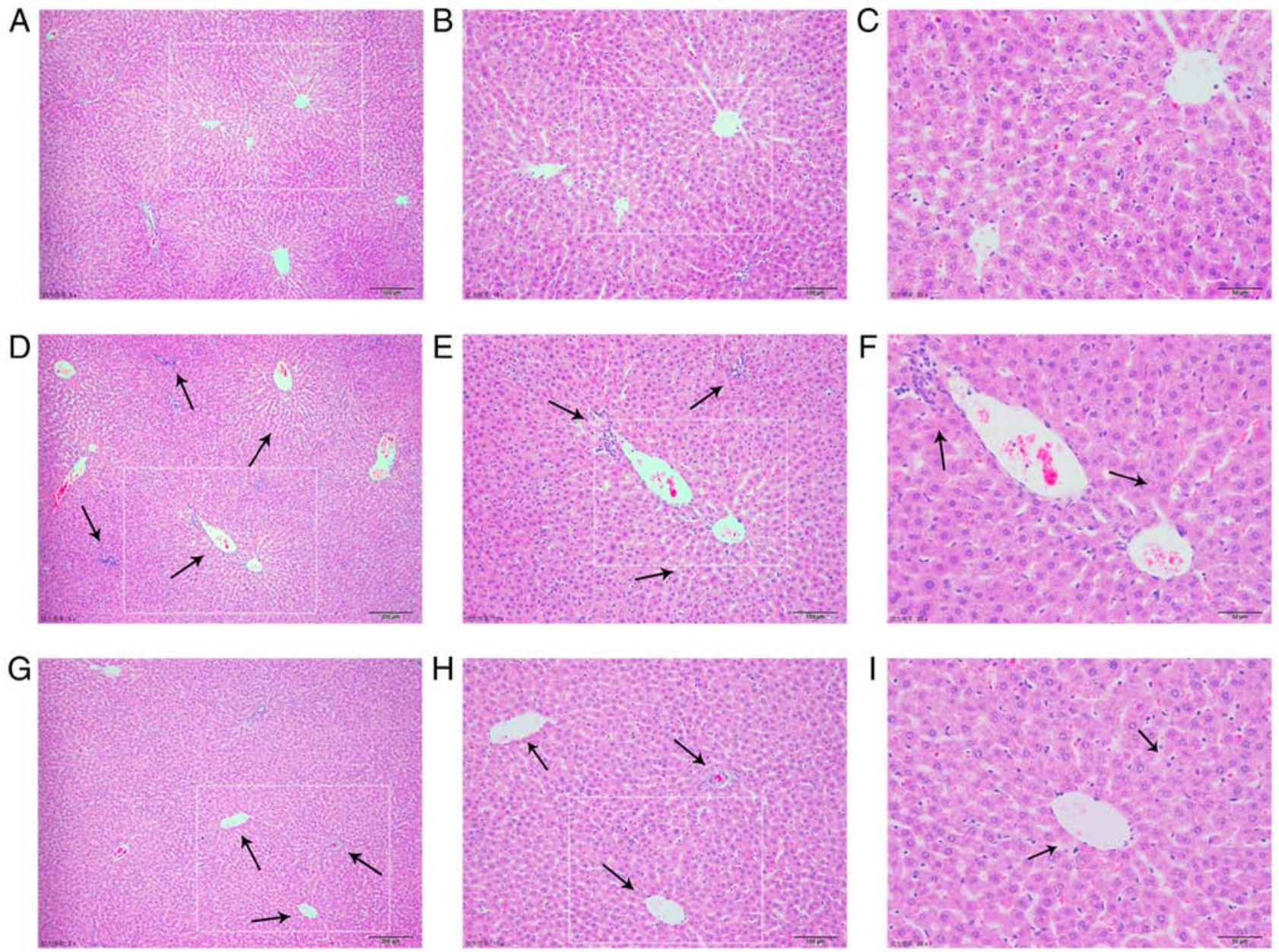

Figure 2. MT reverses histological damage in the liver tissues of rats with $\alpha$-naphthyl isothiocyanate-induced cholestasis. Control group images at magnification of (A) x50, (B) x100 and (C) x200; cholestasis model group images at magnification of (D) x50, (E) x100 and (F) x200; and MT treatment group images at magnification of (G) x50, (H) x100 and (I) x200. Liver tissues histological damage is indicated by black arrows. MT, melatonin.

in Fig. 2. The liver in the control group had a normal lobular architecture with central veins and radiating hepatic cords (Fig. 2A-C). By contrast, rats with cholestasis exhibited marked changes in liver morphology, including acute infiltration of polymorphonuclear neutrophils, fatty metamorphosis, sinusoid congestion and necrotic inflammation (Fig. 2D-F). Rats treated with MT exhibited mild bile duct epithelial damage and hydropic degeneration of hepatocytes with fewer invading neutrophils (Fig. 2G-I).

Multivariate statistical analysis. PCA was conducted to assess the differences in the metabolome profiles of the three groups. A score plot allowed for visualization of observational clusters, which differed significantly between the control, ANIT, MT and QC groups (Fig. 3A). The results of PCA indicated that multivariate statistical analysis was necessary to clarify the differences among the groups.

OPLS-DA was applied to eliminate and classify uncorrelated noise and identify potential biomarkers based on differences in metabolome profiles. The ANIT, MT $(100 \mathrm{mg} / \mathrm{kg})$ and control groups were distinguished in the models (Fig. 3B and C). The $\mathrm{R}^{2} \mathrm{X}, \mathrm{R}^{2} \mathrm{Y}$ and $\mathrm{Q}^{2}$ (cum) of the ANIT and control groups were $0.437,0.996$ and 0.974 respectively, as compared with the values of $0.349,0.983$ and 0.909 respectively which from ANIT group and MT group. These results indicated that the model was of good quality and provided reliable confidence predictions. Permutation tests with 200 iterations and validation plots indicated that the original models were valid (Fig. 3D and E). An analysis of OPLS-DA loading and score plots revealed several critical variables that were far removed from the center of the coordinate of the loading plot (Fig. 3F and G), suggesting that they were important for clustering.

Metabolite identification in MT-treated rats with cholestasis. Metabolites whose concentration varied among groups were selected based on the combination of a statistically significant threshold of VIP values obtained from the OPLS-DA model and P-values from a two-tailed Student's t-test of normalized peak areas. Metabolites with values of VIP $>1$ and $\mathrm{P}<0.05$ were included. The number and trend of metabolites in different groups were presented in Fig. 4. Candidates that significantly differed among the groups with $\mathrm{P}<0.05$ and $\log \mid$ fold changel $>2$ were identified as candidate biomarkers for cholestasis and MT treatment (Table II). Variations in the trends of seven biomarkers, which included 5-aminopentanoate, 5-methoxytryptamine, L-tryptophan, threonine, glutathione (GSH), L-methionine and indolelactate, were presented in 
A

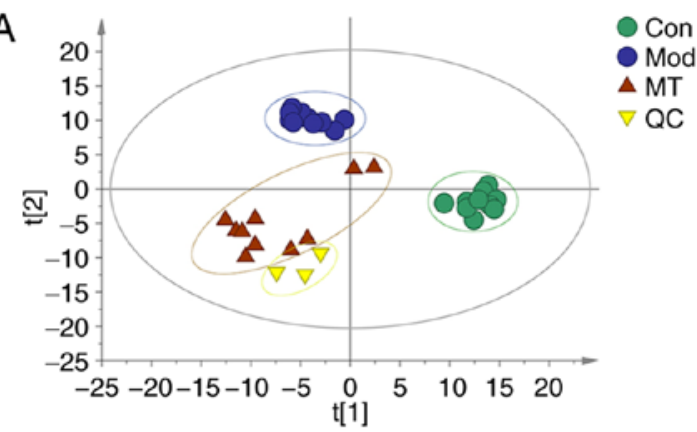

$R 2 \times[1]=0.233 \quad R 2 \times[2]=0.164$ Ellipse: Hotelling's T2 $(95 \%)$

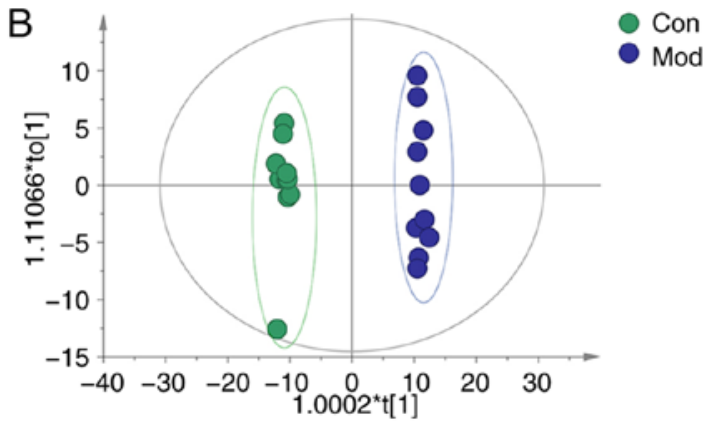

R2×[1] $=0.358 \quad$ R2×o[1] $=0.079$ Ellipse: Hotelling's T2 $(95 \%)$
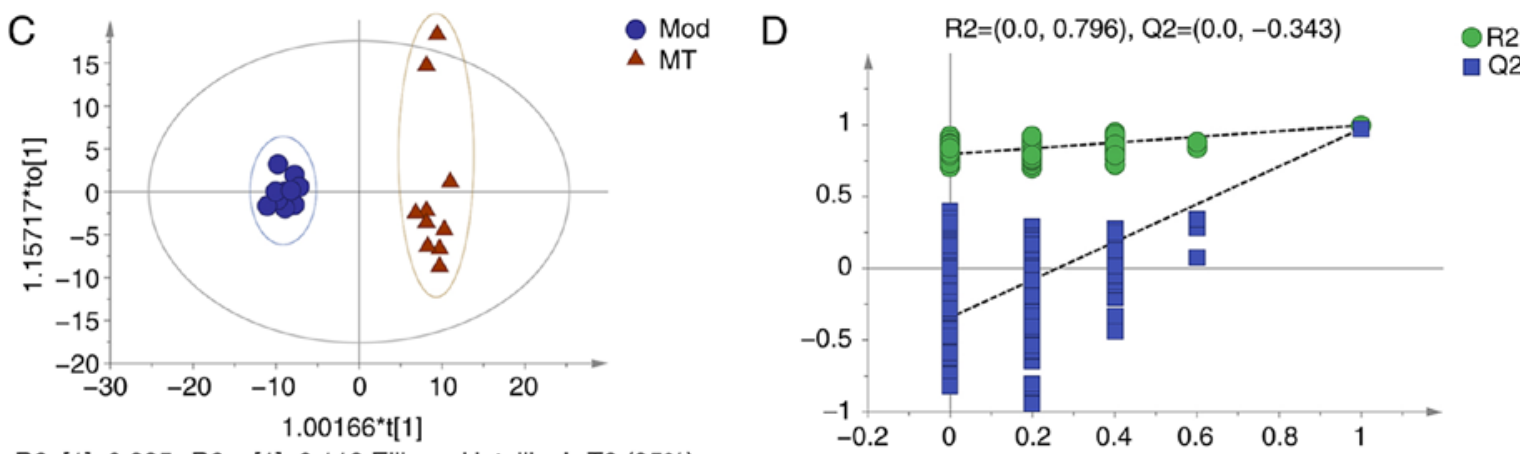

R2×[1] $=0.235$ R2×o[1] $=0.113$ Ellipse: Hotelling's T2 (95\%)
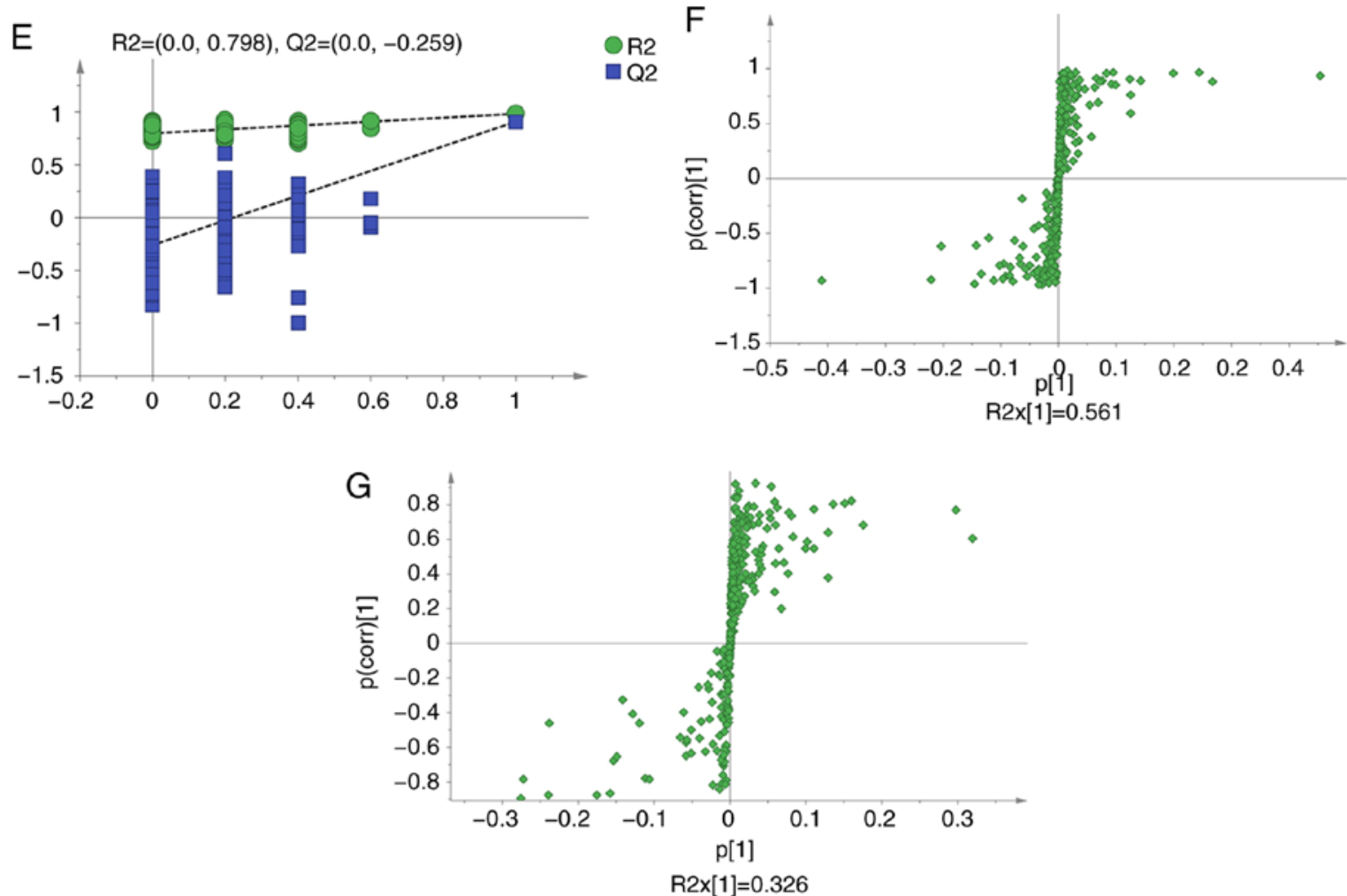

Figure 3. OPLS-DA, 200-permutation test and OPLS-DA score plot of cholestasis rats with or without MT treatment. (A) Principle component analysis score plot of the Con, Mod (ANIT), MT and QC groups. (B) OPLS-DA of Cholestasis model (ANIT) vs. Con group. (C) OPLS-DA of MT treatment vs. ANIT group. (D) 200-permutation test of Cholestasis model (ANIT) and Con group. (E) 200-permutation test of MT treatment and ANIT group. (F) OPLS-DA score plot of Cholestasis model (ANIT) and Con group. (G) OPLS-DA score plot of MT treatment and ANIT group. OPLS-DA, orthogonal partial least-squares discriminant analysis; MT, melatonin; Con, control; Mod, model; QC, quality control; ANIT, $\alpha$-naphthyl isothiocyanate.

Fig. 5. Cholestasis model rats had markedly elevated serum L-tryptophan, L-methionine and threonine levels, as well as significantly lower levels of the other four metabolites, when compared with the control group. By contrast, the MT group exhibited markedly elevated serum 5-methoxytryptamine, indolelactate, GSH and 5-aminopentanoate levels, and reduced levels of the other three metabolites when compared with the model group. 
Table II. Identified metabolites in the serum of the different groups.

\begin{tabular}{|c|c|c|c|c|c|c|c|c|c|c|}
\hline \multirow[b]{3}{*}{ RT (min) } & \multirow{3}{*}{$\begin{array}{c}\text { Mass } \\
(\mathrm{Da})\end{array}$} & \multirow[b]{3}{*}{ Metabolite } & \multirow{2}{*}{\multicolumn{2}{|c|}{ ANOVA analysis }} & \multirow[b]{3}{*}{ VIP } & \multicolumn{2}{|c|}{ Model and control } & \multirow[b]{3}{*}{ VIP } & \multicolumn{2}{|c|}{ MT and model } \\
\hline & & & & & & Fold & P-value of & & Fold & P-value of \\
\hline & & & F-value & P-value & & change & Tukey's test & & change & Tukey's test \\
\hline 13.11 & 174 & 5-Aminopentanoate & 24.711 & $<0.001$ & 1.52 & 0.78 & 0.002 & 1.71 & 1.50 & $<0.001$ \\
\hline 7.41 & 174 & 5-Methoxytryptamine & 29.066 & $<0.001$ & 1.51 & 0.79 & $<0.001$ & 1.21 & 1.10 & 0.029 \\
\hline 28.57 & 202 & Tryptophan & 24.329 & $<0.001$ & 1.44 & 1.75 & $<0.001$ & 1.62 & 0.65 & $<0.001$ \\
\hline 12.60 & 218 & Threonine & 31.933 & $<0.001$ & 1.43 & 1.72 & $<0.001$ & 1.72 & 0.64 & $<0.001$ \\
\hline 5.87 & 107 & Glutathione & 14.065 & $<0.001$ & 1.40 & 0.79 & $<0.001$ & 1.45 & 1.20 & 0.002 \\
\hline 15.42 & 176 & Methionine & 12.485 & $<0.001$ & 1.32 & 1.44 & $<0.001$ & 1.35 & 0.78 & 0.005 \\
\hline 17.04 & 202 & Indolelactate & 11.250 & $<0.001$ & 1.10 & 0.55 & 0.130 & 1.46 & 2.92 & $<0.001$ \\
\hline
\end{tabular}

RT, retention time; MT, melatonin; VIP, variable influence on projection.
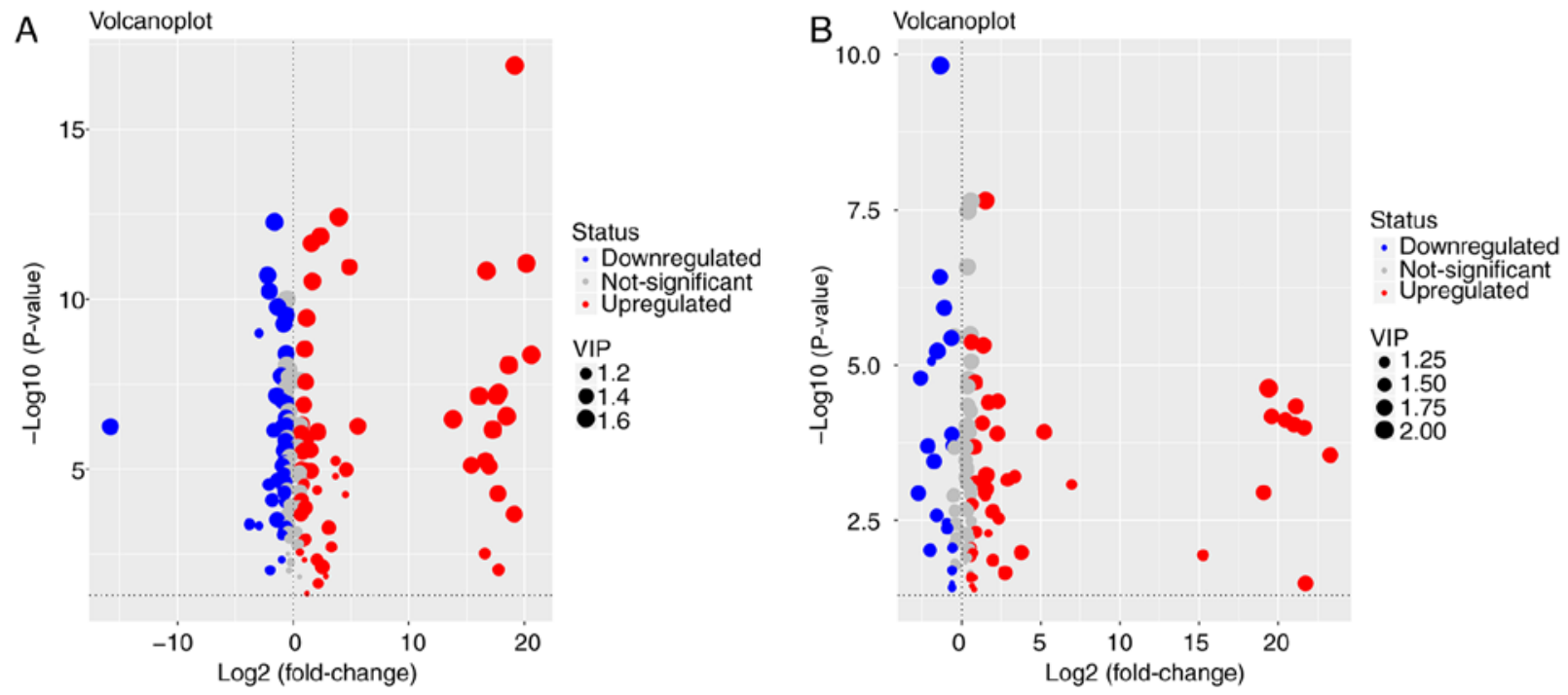

Figure 4. Volcano plot of metabolites in the serum and liver tissue of the (A) cholestasis model ( $\alpha$-naphthyl isothiocyanate-treated) vs. the control group, and (B) melatonin treatment vs. model group. The $\mathrm{y}$-axis shows the- $\log _{10}\left(\mathrm{P}\right.$-value), while the $\mathrm{x}$-axis presents $\log _{2}$ (fold change). Red, blue and grey circles represent the upregulated, downregulated and non-significant changes, respectively, while the size of the circles reflects the VIP value. VIP, variable influence on projection.

Pathway analysis in MT-treated rats with cholestasis. Bubble diagrams of the control, ANIT and MT groups are presented in Fig. 6, where the $\mathrm{x}$-axis shows the Rich factor, which is the ratio of the differential metabolite number to the total number of metabolites in model organisms in each pathway. The color and size of each circle reflect the P-value and the variation in the metabolite number in each pathway, respectively. The results revealed that the seven metabolites with a significantly different expression among the groups appeared in the following four KEGG pathways: i) Glycine, serine and threonine metabolism; ii) cysteine and methionine metabolism; iii) tryptophan metabolism; and iv) arginine and proline metabolism. Specifically, those associated with amino acids were focused on.

\section{Discussion}

Cholestasis is characterized by intrahepatic accumulation of potentially toxic bile acids resulting from hepatocellular dysfunction or bile duct obstruction (2). However, the detailed mechanism underlying this condition has not been fully elucidated. Identifying sensitive and specific biomarkers is important for the early detection of hepatic fibrosis. In the present study, the GC-MS-based serum metabolomics analysis revealed significant changes in pathways associated with the metabolism of the amino acids arginine/proline, glycine/serine/threonine, tryptophan and cysteine/methionine. Significant differences were also observed in the levels of 5-aminopentanoate, 5-methoxytryptamine, L-tryptophan, threonine, glutathione (GSH), L-methionine and indolelactate among the control rats and the cholestasis model rats with or without MT treatment.

Based on the KEGG database results, L-tryptophan, 5-methoxytryptamine and indolelactate in the serum are by-products of tryptophan metabolism, while GSH and L-methionine are by-products of the cysteine and methionine metabolism. In addition, threonine is a by-product of the glycine, serine and threonine metabolism, and 5-aminopentanoate is 
A

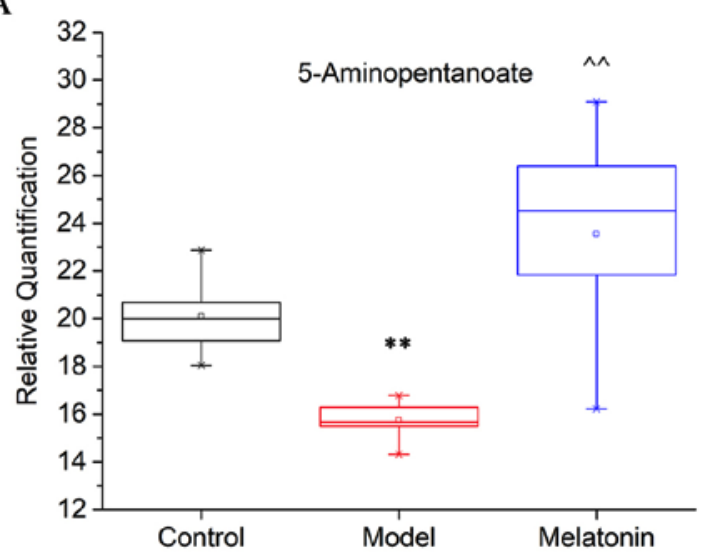

C

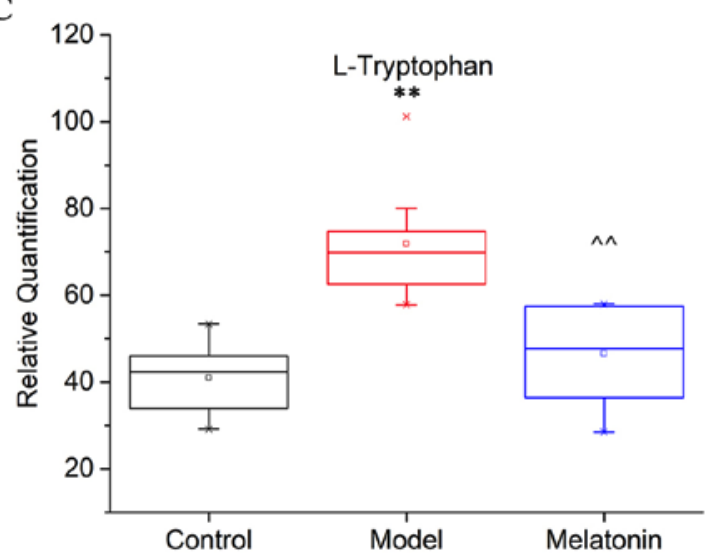

E

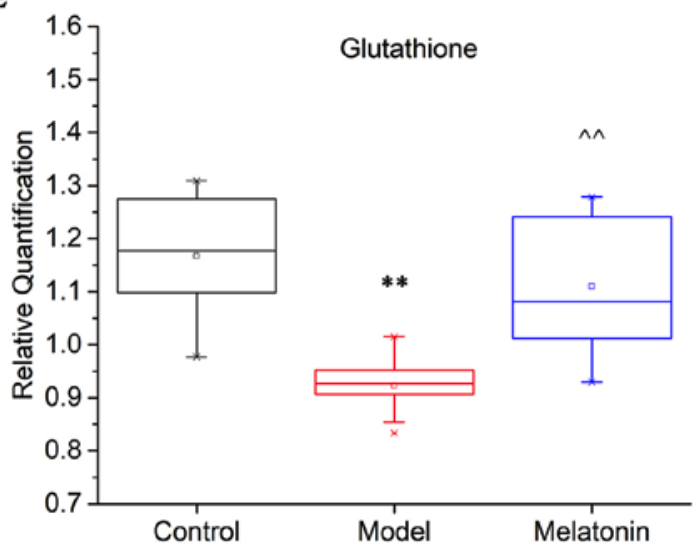

B

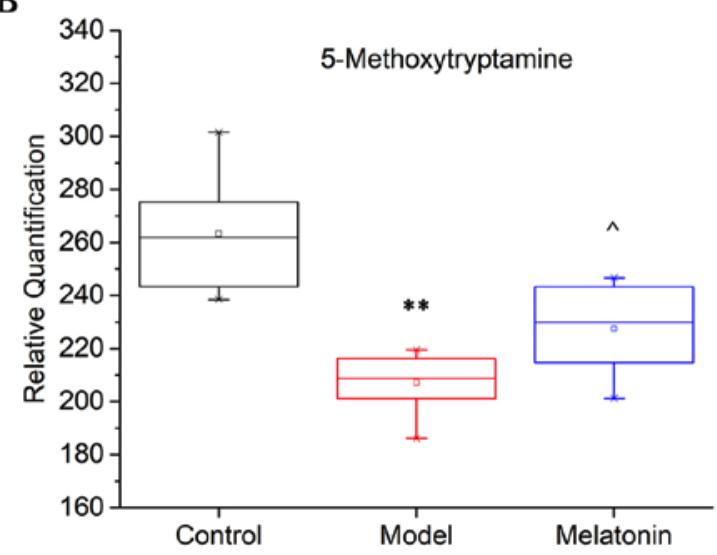

D

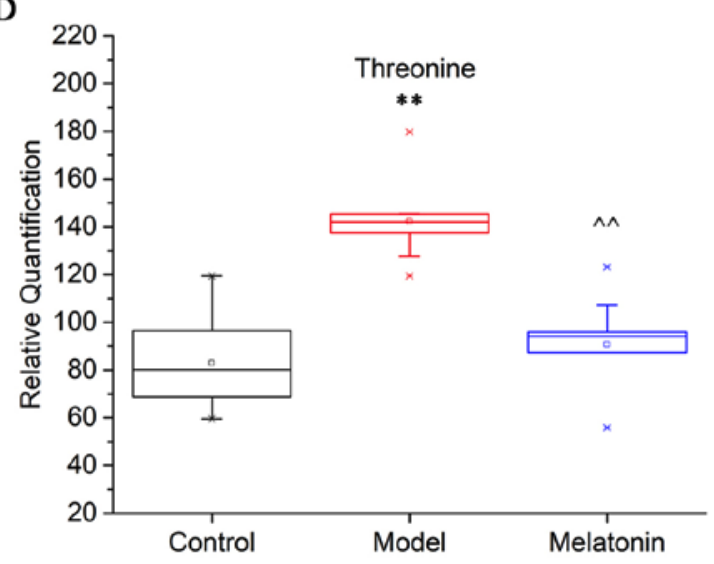

F

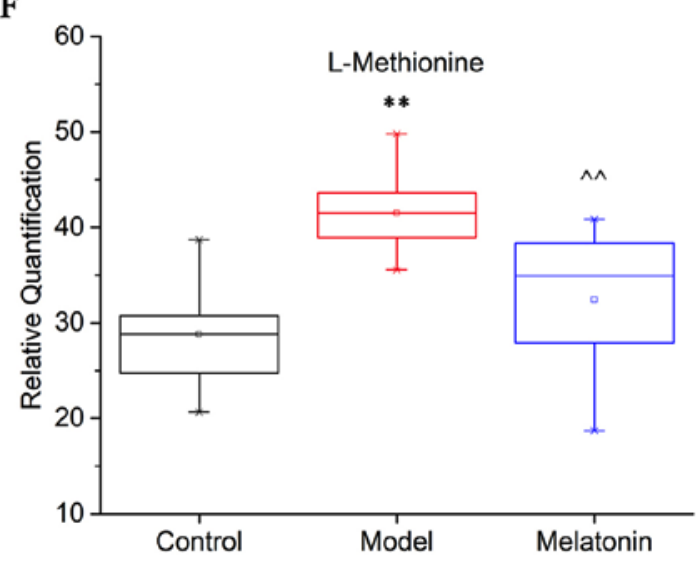

\section{G}

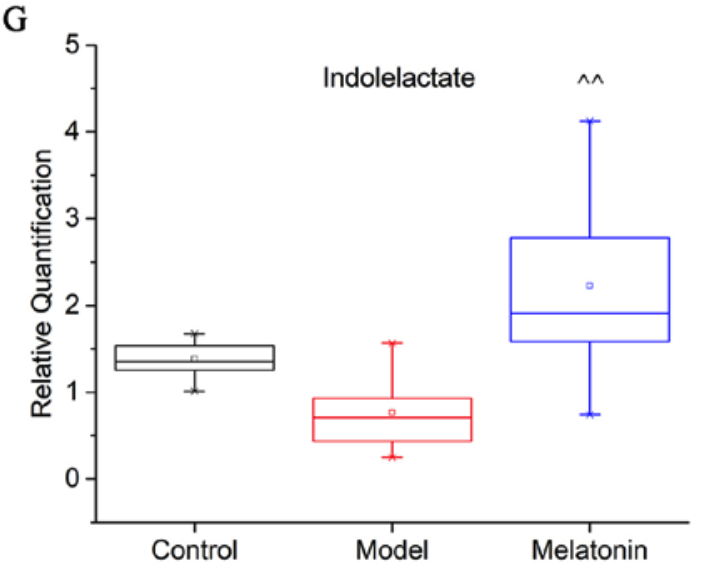

Figure 5. Changes in metabolome profiles in the cholestasis rats with or without melatonin treatment. The levels of (A) 5-aminopentanoate, (B) 5-methoxytryptamine, (C) L-tryptophan, (D) threonine, (E) glutathione, (F) L-methionine, and (G) indolelactate are displayed. The squares indicate the mean value of expression. ${ }^{* *} \mathrm{P}<0.01$ vs. control group; ${ }^{\wedge} \mathrm{P}<0.05$ and ${ }^{\wedge \wedge} \mathrm{P}<0.01$ vs. cholestasis model group. 
A

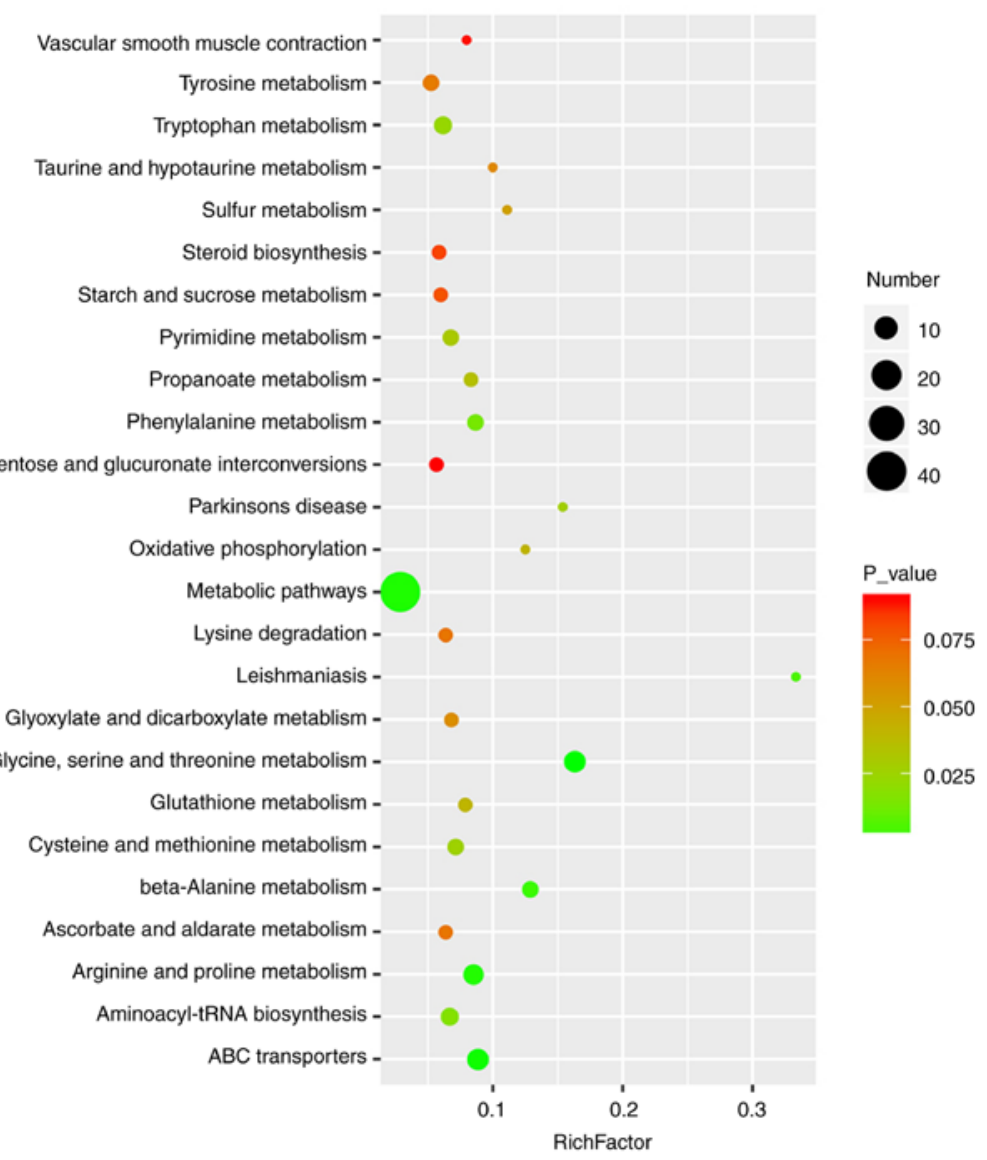

B
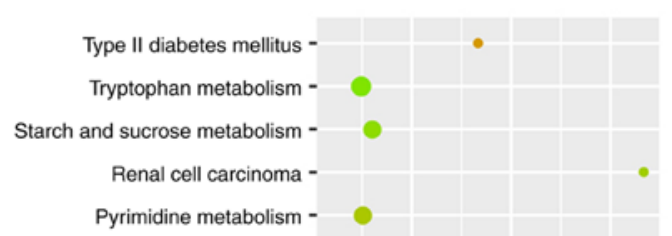

产

Inositol phosphate metabolism -

Glyoxylate and dicarbooxylate metabolism Glycine, serine and threonine metabolism -

Glutathione metabolism -

Galactose metabolism -

Fatty acid biosynthesis -

Cysteine and methionine metabolism -

Cyanoamino acid metabolism -

Biosynthesis of unsaturated fatty acids -

beta-Alanine metabolism -

Ascorbate and aldarate metabolism -

Arginine and proline metabolism -

Aminoacyl-tRNA biosynthesis -

$\mathrm{ABC}$ transportetrs -

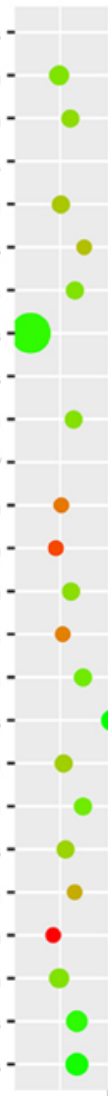




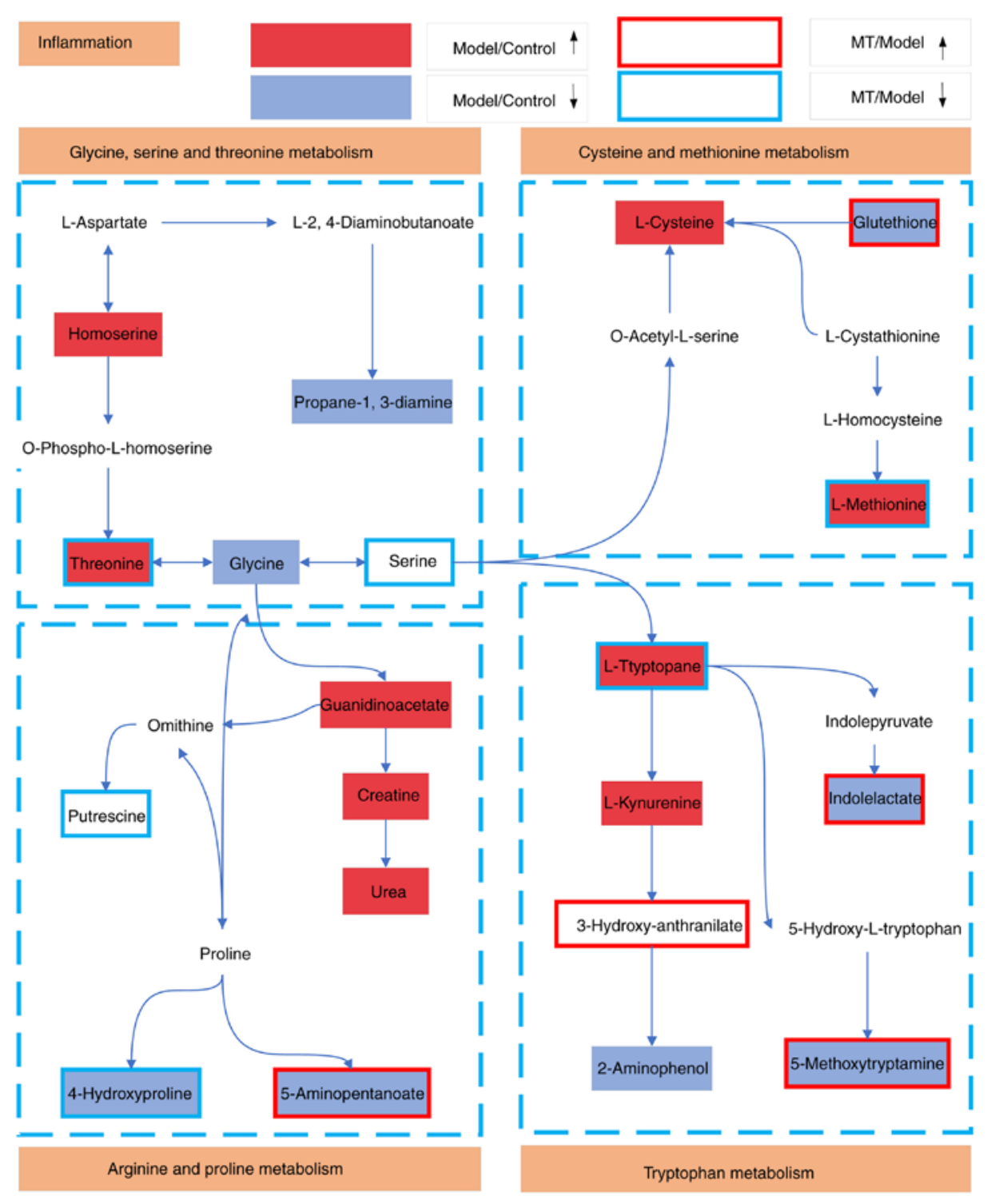

Figure 7. Schematic illustration of metabolic pathways associated with cholestasis that were altered by MT treatment. Red and light blue boxes indicate metabolites with significantly higher and lower levels, respectively, in the cholestasis model when compared with the control group. Boxes bordered in red and blue represent metabolites with significantly higher and lower levels, respectively, in the MT treatment group when compared with the control rats. MT, melatonin.

a by-product of the arginine and proline metabolism. Levels of these metabolites were also demonstrated to be altered in serum. These results suggested that the metabolism of arginine, proline, glycine, serine, threonine, tryptophan, cysteine and methionine may be disrupted in cholestasis (Fig. 7).

GSH is a thiol compound that is associated with catabolism and intracellular transport. Alterations in plasma GSH levels are associated with Parkinson's and Alzheimer's diseases, diabetes, macular degeneration and human immunodeficiency virus infection (36). GSH participates in a number of cellular functions, including protection from free radicals during oxidative stress (37). It has been reported that, for partial hepatectomy in rats, hepatic GSH levels have increased after $24 \mathrm{~h}(38,39)$. however, due to the short half-life of GSH, liver GSH levels return to baseline despite this elevation within $48 \mathrm{~h}$ (40). 5-Methoxytryptamine is the main metabolite produced in the transformation of MT by MT deacetylase, which is subsequently metabolized to 5-methoxyindoleacetaldehyde, 5-methoxyindole acetic acid or 5-methoxytryptophol (41). A previous study reported that only a small fraction (approximately $0.3-0.8 \%$ ) of the concentration of the MT present in the incubation medium was converted to 5-MT (42). This indicates that deacetylation of MT is one possible pathway for the biosynthesis of 5-MT. Indolepropionic acid is a product of the microbial degradation of L-tryptophan (43). MT and indolepropionic acid function as endogenous electron donors, primarily detoxifying reactive radicals; however, they do not undergo autooxidation in the presence of transition metals (44). Furthermore, previous results have indicated that indolepropionic acid is effective in protecting rat hepatic microsomal membranes against rigidity and against lipid peroxidation caused by iron (45). Additionally, 5 -aminopentanoate has been reported to serve an important role in proline metabolism and may participate in lysine degradation in Phaeobacter inhibens DSM 17395 (46). Lee et al (47) also found that lysine degradation is specifically associated with stroke occurrence, and that low expression of 5-aminopentanoate may increase the risk of thrombotic stroke. In the present study, serum GSH, 
5-methoxytryptamine, indolepropionic and 5-aminopentanoate levels were decreased in cholestasis model rats when compared with the control rats; however, these level were increased by MT treatment. Thus, these four metabolites may be useful biomarkers for diagnosing cholestasis and evaluating responses to MT treatment.

L-Tryptophan, a precursor of serotonin and MT, serves a role in depression, schizophrenia and somatization (48). Lower levels of plasma tryptophan are associated with enhanced pain, autonomic nervous system responses, gut motility, peripheral nerve function, and ventilation and cardiac dysfunction $(49,50)$. Threonine is an essential amino acid that is incorporated into intestinal mucosal proteins and is required for the synthesis of secretory glycoproteins (51). Threonine and alanine share the same amino acid transporter, which is responsible for threonine uptake into different cell types, including hepatocytes (52). This is the rate-limiting step for threonine utilization by the liver $(53,54)$. Methionine is a metabolite that is important for GSH synthesis in the liver (55). Oral administration of methionine at high doses was reported to markedly elevate the level of homocysteine in rat plasma, while long-term MT administration significantly reduced homocysteine levels (56). Based on this observation, the close association between methionine and GSH is confirmed. Furthermore, homocysteine may be the intermediate metabolite between MT and methionine. In the present study, serum L-tryptophan levels were increased in the cholestasis model rats; however, this was abolished by MT treatment, suggesting that L-tryptophan may be associated with MT secretion and, thus, may serve as a biomarker in cholestasis.

In conclusion, the results of the present study demonstrated that MT has a significant anti-cholestatic effect. Seven metabolites, including 5-aminopentanoate, 5-methoxytryptamine, L-tryptophan, threonine, GSH, L-methionine and indolelactate, were identified as potential biomarkers of cholestasis, which may be useful for disease diagnosis and for assessing the therapeutic efficacy of MT treatment.

\section{Acknowledgements}

The authors would like to thank the staff of the Science Center Department of Beijing University of Chinese Medicine (Beijing, China), the Pathology Department of China-Japan Friendship Hospital (Beijing, China), Shanghai OE Biotech, Inc. (Shanghai, China) and Professor Jian Li and Mrs. Shujing Zhang for their technical support.

\section{Funding}

Not applicable.

\section{Availability of data and materials}

All data generated or analyzed during this study are included in this published article.

\section{Authors' contributions}

HY, YL and ZX contributed equally to the present study. XZ and XD conceived and designed the study. HY, YL and ZX acquired, analyzed and interpreted the data. DW and SS were responsible for handling the animals and obtaining tissue specimens. HD and BZ performed histopathological analyses. ZZ and LS performed statistical analyses. All authors approved the final manuscript.

\section{Ethics approval and consent to participate}

The study protocol was in strict accordance with the recommendations of the Guidelines for the Care and Use of Laboratory Animals of the Ministry of Science and Technology of China, and was approved by Beijing University of Chinese Medicine Medical and Experimental Animal Ethics Committee (Beijing, China).

\section{Patient consent for publication}

Not applicable.

\section{Competing interests}

The authors declare that they have no competing interests.

\section{References}

1. Cao X, Gao Y, Zhang W, Xu P, Fu Q, Chen C, Li C, Yang C, $\mathrm{Ma} \mathrm{G}, \mathrm{Qu}$ Y, et al: Cholestasis morbidity rate in first-hospitalized patients with chronic liver disease in Shanghai. Zhonghua Gan Zang Bing Za Zhi 23: 569-573, 2015 (In Chinese).

2. Yang K, Köck K, Sedykh A, Tropsha A and Brouwer KL: An updated review on drug-induced cholestasis: Mechanisms and investigation of physicochemical properties and pharmacokinetic parameters. J Pharm Sci 102: 3037-3057, 2013.

3. European Association for the Study of the Liver: EASL clinical practice guidelines: Management of cholestatic liver diseases. J Hepatol 51: 237-267, 2009.

4. Park HW, Lee NM, Kim JH, Kim KS and Kim SN: Parenteral fish oil-containing lipid emulsions may reverse parenteral nutrition-associated cholestasis in neonates: A systematic review and meta-analysis. J Nutr 145: 277-283, 2015.

5. Boyer JL: New perspectives for the treatment of cholestasis: Lessons from basic science applied clinically. J Hepatol 46: 365-371, 2007.

6. Pollock G and Minuk GY: Diagnostic considerations for cholestatic liver disease. J Gastroenterol Hepatol 32: 1303-1309, 2017.

7. Allen K, Jaeschke H and Copple BL: Bile acids induce inflammatory genes in hepatocytes: A novel mechanism of inflammation during obstructive cholestasis. Am J Pathol 178: 175-186, 2011.

8. Weerachayaphorn J, Luo Y, Mennone A, Soroka CJ, Harry K and Boyer JL: Deleterious effect of oltipraz on extrahepatic cholestasis in bile duct-ligated mice. J Hepatol 60: 160-166, 2014.

9. Beuers U, Rauner M, Jansen P and Poupon R: New paradigms in the treatment of hepatic cholestasis: From UDCA to FXR, PXR and beyond. J Hepatol 62 (Suppl 1): S25-S37, 2015.

10. Kaplan MM and Gershwin ME: Primary biliary cirrhosis. N Engl J Med 353: 1261-1273, 2005.

11. McKiernan PJ: Neonatal cholestasis. Semin Neonatol 7: 153-165, 2002.

12. Ghonem NS, Assis DN and Boyer JL: Fibrates and cholestasis. Hepatology 62: 635-643, 2015.

13. Acuña-Castroviejo D, Escames G, Venegas C, Díaz-Casado ME Lima-Cabello E, López LC, Rosales-Corral S, Tan DX and Reiter RJ: Extrapineal melatonin: Sources, regulation, and potential functions. Cell Mol Life Sci 71: 2997-3025, 2014.

14. Pääkkönen T, Mäkinen TM, Leppäluoto J, Vakkuri O, Rintamäki H, Palinkas LA and Hassi J: Urinary melatonin: A noninvasive method to follow human pineal function as studied in three experimental conditions. J Pineal Res 40: 110-115, 2006.

15. Claustrat B and Leston J: Melatonin: Physiological effects in humans. Neurochirurgie 61: 77-84, 2015.

16. Calvo JR, González-Yanes C and Maldonado MD: The role of melatonin in the cells of the innate immunity: A review. J Pineal Res 55: 103-120, 2013. 
17. Lotufo CM, Lopes C, Dubocovich ML, Farsky SH and Markus RP: Melatonin and N-acetylserotonin inhibit leukocyte rolling and adhesion to rat microcirculation. Eur J Pharmacol 430: 351-357, 2001.

18. Allegra M, Reiter RJ, Tan DX, Gentile C, Tesoriere L and Livrea MA: The chemistry of melatonin's interaction with reactive species. J Pineal Res 34: 1-10, 2003.

19. Jaworek J, Leja-Szpak A, Kot M, Jaworek A, Nawrot-Porbka K, Bonior J and Szklarczyk J: The role of melatonin in pancreatic protection: Could melatonin be used in the treatment of acute pancreatitis? Curr Pharm Des 20: 4834-4840, 2014.

20. Ohta Y, Kongo-Nishimura M, Imai Y and Kitagawa A: Melatonin attenuates disruption of serum cholesterol status in rats with a single alpha-naphthylisothiocyanate treatment. J Pineal Res 42 : 159-165, 2007.

21. Wang JB, Zhao HP, Zhao YL, Jin C, Liu DJ, Kong WJ, Fang F, Zhang L, Wang HJ and Xiao XH: Hepatotoxicity or hepatoprotection? Pattern recognition for the paradoxical effect of the Chinese herb Rheum palmatum L. in treating rat liver injury. PLoS One 6: e24498, 2011

22. Wang X, Zhang A, Han Y, Wang P, Sun H, Song G, Dong T, Yuan Y, Yuan X, Zhang M, et al: Urine metabolomics analysis for biomarker discovery and detection of jaundice syndrome in patients with liver disease. Mol Cell Proteomics 11: 370-380, 2012.

23. Ma X, Chi YH, Niu M, Zhu Y, Zhao YL, Chen Z, Wang JB, Zhang CE, Li JY, Wang LF, et al: Metabolomics coupled with multivariate data and pathway analysis on potential biomarkers in cholestasis and intervention effect of Paeonia lactiflora Pall. Front Pharmacol 7: 14, 2016.

24. Sun H, Zhang AH, Zou DX, Sun WJ, Wu XH and Wang XJ: Metabolomics coupled with pattern recognition and pathway analysis on potential biomarkers in liver injury and hepatoprotective effects of yinchenhao. Appl Biochem Biotechnol 173: 857-869, 2014

25. Zhang CE, Niu M, Li RY, Feng WW, Ma X, Dong Q, Ma ZJ, Li GQ, Meng YK, Wang Y, et al: Untargeted metabolomics reveals dose-response characteristics for effect of rhubarb in a rat model of cholestasis. Front Pharmacol 7: 85, 2016.

26. Hill DA and Roth RA: Alpha-naphthylisothiocyanate causes neutrophils to release factors that are cytotoxic to hepatocytes. Toxicol Appl Pharmacol 148: 169-175, 1998.

27. Hill DA, Jean PA and Roth RA: Bile duct epithelial cells exposed to alpha-naphthylisothiocyanate produces a factor that causes neutrophil-dependent hepatocellular injury in vitro. Toxicol Sci 47: 118-125, 1999.

28. Cullen JM, Faiola B, Melich DH, Peterson RA, Jordan HL, Kimbrough CL and Miller RT: Acute alpha-naphthylisothiocyanate-induced liver toxicity in germfree and conventional male rats. Toxicol Pathol 44: 987-997, 2016

29. Li X, Liu R, Yu L, Yuan Z, Sun R, Yang H, Zhang L and Jiang Z: Alpha-naphthylisothiocyanate impairs bile acid homeostasis through AMPK-FXR pathways in rat primary hepatocytes. Toxicology 370: 106-115, 2016

30. Golbar HM, Izawa T, Wijesundera KK, Bondoc A, Tennakoon AH, Kuwamura M and Yamate J: Depletion of hepatic macrophages aggravates liver lesion induced in rats by thioacetamide (TAA). Toxicol Pathol 44: 246-258, 2016.

31. Capizzo F and Roberts RJ: $\alpha$-Naphthylisothiocyanate (ANIT)induced hepatotoxicity and disposition in various species. Toxicol Appl Pharmacol 19: 176-187, 1971.

32. Plaa GL and Priestly BG: Intrahepatic cholestasis induced by drugs and chemicals. Pharmacol Rev 28: 207-273, 1976.

33. Cruz A,PadilloFJ, Torres E, Navarrete CM, Muñoz-Castañeda JR, Caballero FJ, Briceño J, Marchal T, Túnez I, Montilla P, et al: Melatonin prevents experimental liver cirrhosis induced by thioacetamide in rats. J Pineal Res 39: 143-150, 2005.

34. Bothe MK, Meyer C, Mueller U, Queudot JC, Roger V, Harleman J and Westphal M: Characterization of a rat model of moderate liver dysfunction based on alpha-naphthylisothiocyanate-induced cholestasis. J Toxicol Sci 42: 715-721, 2017.

35. Zhou HQ, Liu W, Wang J, Huang YQ, Li PY, Zhu Y, Wang JB, Ma X, Li RS, Wei SZ, et al: Paeoniflorin attenuates ANIT-induced cholestasis by inhibiting apoptosis in vivo via mitochondria-dependent pathway. Biomed Pharmacother 89 696-704, 2017.

36. Yuan B, Xu C, Liu L, Zhang Q, Ji S, Pi L, Zhang D and Huo Q: $\mathrm{Cu}_{2} \mathrm{O} / \mathrm{NiO}_{\mathrm{x}} /$ graphene oxide modified glassy carbon electrode for the enhanced electrochemical oxidation of reduced glutathione and nonenzyme glucose sensor. Electrochimica Acta 104: 78-83, 2013.
37. Jaeschke H: The role of reactive oxygen species in hepatic ischaemia-reperfusion injury and preconditioning. J Inv Surg 16: 127-140, 2003.

38. Carbonell LF, Nadal JA, Llanos MC, Hernández I, Nava E and Díaz J: Depletion of liver glutathione potentiates the oxidative stress and decreases nitric oxide synthesis in a rat endotoxin shock model. Crit Care Med 28: 2002-2006, 2000.

39. Lee SJ and Boyer TD: The effect of hepatic regeneration on the expression of the glutathione S-transferases. Biochem J 293: $137-142,1993$.

40. Huang ZZ, Li H, Cai J, Kuhlenkamp J, Kaplowitz N and Lu SC: Changes in glutathione homeostasis during liver regeneration in the rat. Hepatology 27: 147-153, 1998.

41. Grace MS, Cahill GM and Besharse JC: Melatonin deacetylation: Retinal vertebrate class distribution and Xenopus laevis tissue distribution. Brain Res 559: 56-63, 1991.

42. Rogawski MA, Roth RH and Aghajanian GK: Melatonin: Deacetylation to 5-methoxytryptamine by liver but not brain aryl acylamidase. J Neurorhem 32, 1219-1226, 1979.

43. Jean M and DeMoss RD: Indolelactate dehydrogenase from Clostridium sporogenes. Can J Microbiol 14: 429-435, 1968.

44. Tan DX, Manchester LC, Reiter RJ, Qi W, Karbownik M and Calvo JR: Significance of melatonin in antioxidative defense: Reactions and products. Biol Signals Recept 9: 137-159, 2000.

45. Karbownik M, Reiter RJ, Garcia JJ, Cabrera J, Burkhardt S, Osuna C and Lewiński A: Indole-3-propionic acid, a melatonin-related molecule, protects hepatic microsomal membranes from ironinduced oxidative damage: Relevance to cancer reduction. J Cell Biochem 81: 507-513, 2001.

46. Drüppel K, Hensler M, Trautwein K, Kozzmehl S, Wöhlbrand L, Schmidt-Hohagen K, Ulbrich M, Bergen N, Meier-Kolthoff JP, Göker $\mathrm{M}$, et al: Pathways and substrate-specific regulation of amino acid degradation in Phaeobacter inhibens DSM 17395 (archetype of the marine Roseobacter clade). Environ Microbiol 16: 218-238, 2014.

47. Lee Y, Khan A, Hong S, Jee SH and Park YH: A metabolomic study on high-risk stroke patients determines low levels of serum lysine metabolites: A retrospective cohort study. Mol Biosyst 1109-1120, 2017.

48. Young SN: The effect of raising and lowering tryptophan levels on human mood and social behaviour. Philos Trans R Soc Lond B Biol Sci 368: 20110375, 2013.

49. Anderson G, Maes M and Berk M: Schizophrenia is primed for an increased expression of depression through activation of immuno-inflammatory, oxidative and nitrosative stress, and tryptophan catabolite pathways. Prog Neuropsychopharmacol Biol Psychiatry 42: 101-114, 2013.

50. Anderson G, Maes M and Berk M: Inflammation-related disorders in the tryptophan catabolite pathway in depression and somatization. Adv Protein Chem Struct Biol 88: 27-48, 2012.

51. Hogewind-Schoonenboom JE, Huang L, de Groof F, Zhu L, Voortman GJ, Schierbeek H, Vermes A, Chen C, Huang Y and van Goudoever JB: Threonine requirement of the enterally fed term infant in the first month of life. J Pediatr Gastroenterol Nutr 61: 373-379, 2015.

52. Kilberg MS, Handlogten ME and Christensen HN: Characteristics of system ASC for transport of neutral amino acids in the isolated rat hepatocyte. J Biol Chem 256: 3304-3312, 1981.

53. Floc'h NL, Obled $C$ and Sève B: In vivo threonine oxidation in growing pigs fed on diets with graded levels of threonine. Br J Nutr 75: 825-837, 1996.

54. Le Floc'h N, Thibault JN and Sève B: Tissue localization of threonine oxidation in pigs. Br J Nutr 77: 593-603, 1997.

55. Lu SC, Mato JM, Espinosa-Diez C and Lamas S: MicroRNA-mediated regulation of glutathione and methionine metabolism and its relevance for liver disease. Free Radic Biol Med 100: 66-72, 2016

56. Bouzouf M, Martinez-Cruz F, Molinero P, Guerrero JM and Osuna C: Melatonin prevents hyperhomocysteinemia and neural lipid peroxidation induced by methionine intake. Curr Neurovasc Res 2: 175-178, 2005.

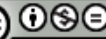

This work is licensed under a Creative Commons Attribution-NonCommercial-NoDerivatives 4.0 International (CC BY-NC-ND 4.0) License. 